\title{
A case of hereditary spastic paraplegia with demyelinating polyneuropathy
}

\section{AML Beligaswatte ${ }^{1}$, S Bandusena ${ }^{2}$, MTM Riffsy ${ }^{3}$, S Gunasekera $^{4}$ and JC Wijesekera ${ }^{5}$}

(Index words: Absent sensory action potentials markedly prolonged distal motor latencies, reduced nerve conduction velocities)

\section{Introduction}

The hereditary spastic paraplegias are a group of heterogeneous disorders characterised by lower limb spasticity. Although most cases represent a 'pure' form, variants with additional 'complicating' features have been documented [1]. We report a patient with hereditary spastic paraplegia (HSP) having a demyelinating form of polyneuropathy which has not been previously associated with this condition.

\section{Case report}

A 14-year old girl presented with difficulty in walking, dysarthria, and learning disability since infancy. A delay of motor milestones had been noticed from the age of 3 months. By the age of 10 years she had a stiff unsteady gait with a tendency to trip, was dysarthric, and had severely impaired school performance. Her symptoms had progressed slowly since then, but there had been no regression of milestones.

Her parents' marriage was non-consanguineous and her antenatal period was uneventful. Her 10-year old brother has a similar illness with a stiff, unsteady gait, dysarthria, and a learning disability, but is less severely affected. Other family members were reportedly normal.

Examination revealed no dysmorphic features or thickened nerves. The abdomen and cardio-respiratory systems were normal. She had bilateral foot drop, pes cavus deformities, and early wasting of the peroneal muscles. There was no wasting of the upper limbs. Her gait was spastic and ataxic.

She had no focal cortical signs. Visual acuity and fields were normal, but the left optic disc was pale. The other cranial nerves were normal. Grade I horizontal nystagmus and mild dysdiadochokinesia were noted. There were no extrapyramidal signs or sensory deficits.

Upper limb tone and reflexes were normal. Muscle power was normal proximally and of MRC grade $4+/ 5$ in the hands. Both lower limbs were very spastic. Knee jerks were brisk but without clonus. The ankle jerks were exaggerated but less so than the knee jerks. Plantar responses were extensor. Muscle power was grade $4 / 5$ proximally and grade $4 / 5$ distally.

${ }^{1}$ Registrar, ${ }^{2}$ Senior Registrar, ${ }^{3}$ Resident Neurologist, ${ }^{4}$ Neurophysiologist, and ${ }^{5}$ Neurologist, Institute of Neurology, National Hospital of Sri Lanka, Colombo.

Correspondence: AB, e-mail: <ashankab@sltnet.lk>. (Competing interests: none declared). Received 3 May 2005 and accepted 5 July 2005. 


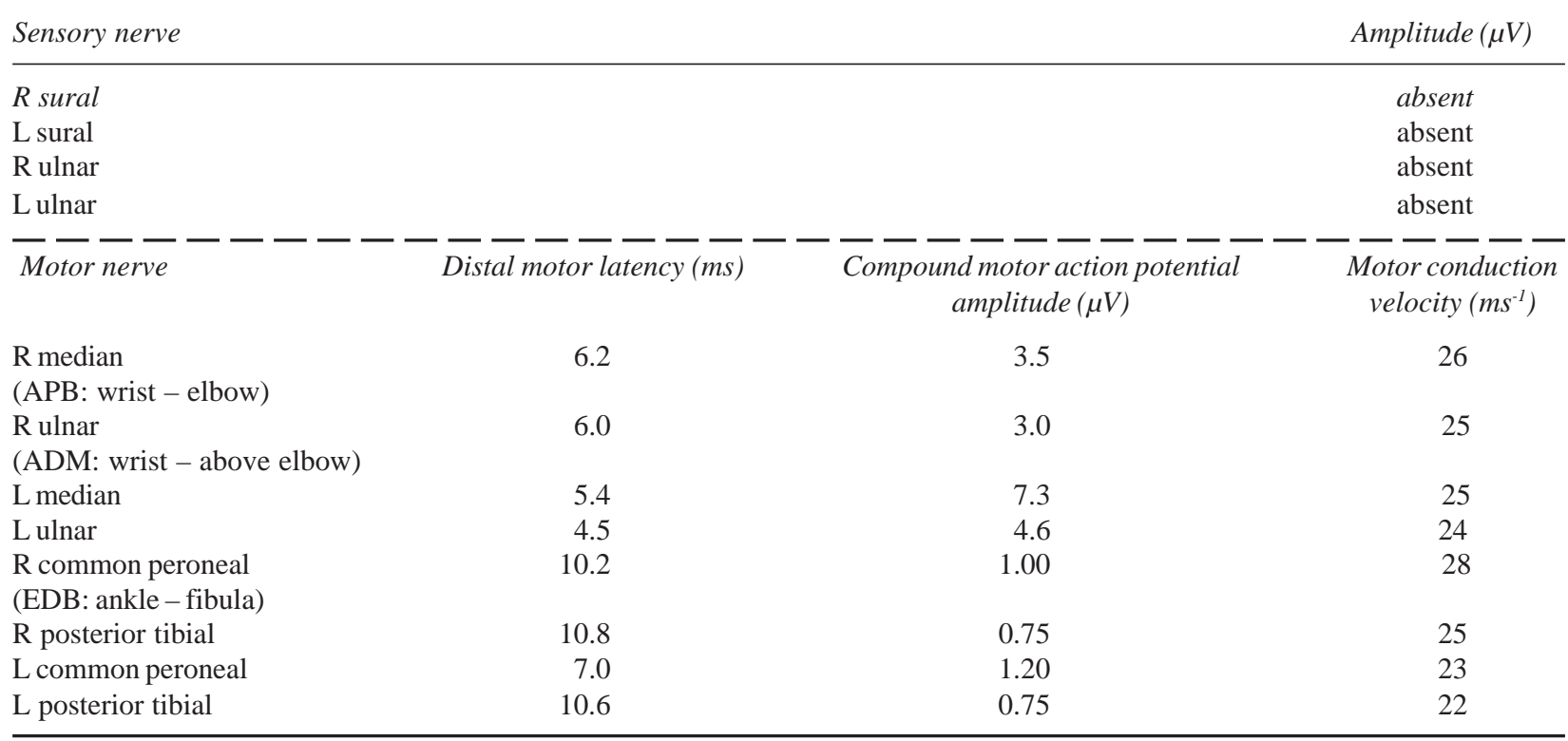

$\mathrm{ADM}=$ abductor digiti minimi, $\mathrm{APB}=$ abductor pollicis brevis, $\mathrm{EDM}=$ extensor digitorum brevis, $\mathrm{R}=$ right, $\mathrm{L}=$ left.

Cerebrospinal fluid had $64 \mathrm{mg} / \mathrm{dL}$ of proteins but was otherwise normal. Electroencephalography and magnetic resonance imaging scans of the head and cervico-dorsal spine revealed no abnormalities. In view of the clinical features suggestive of peripheral neuropathy a nerve conduction study (NCS) was performed, and the findings are shown in Table 1. Visual evoked potential testing showed prolonged $\mathrm{P}_{100}$ latencies with reduced amplitudes bilaterally. The patient's brother had a normal NCS and a normal computerised tomography (CT) scan of the head.

\section{Discussion}

Spasticity of the lower limbs dominated the clinical presentation of both siblings. Their illness is best classified as a hereditary spastic paraplegia. The presence of mild cerebellar signs and learning disability indicates that both siblings have a 'complicated' HSP variant. The sister, who was more severely affected, has in addition, optic nerve atrophy and a peripheral neuropathy. Such phenotypic variation, even within a kindred, is a feature of HSP [1].

Hereditary motor and sensory neuropathy (HMSN) with pyramidal signs, designated HMSN type V, could also possibly account for the patient's presentation. However, it is considered a distinct entity associated with prominent peroneal muscular atrophy and an axonal neuropathy $[2$, $3]$. The relatively mild cerebellar signs argued against a diagnosis of a hereditary ataxia. An inherited infantile leucodystrophy was unlikely given the long survival and lack of white matter changes on neuroimaging.

Variants of HSP with distal amyotrophy, e.g. the Troyer and Silver syndromes, have been described, but in them the wasting is prominent in the hands $[4,5]$. Polyneuropathy reported up to date in HSP is of a sensory axonal type and often results in mutilation of the extremities [6]. In this patient, the marked prolongation of distal motor latencies and severely reduced nerve conduction velocities are typical of a demyelinating motor neuropathy. Absence of sensory action potentials indicates involvement of sensory fibres as well. There are no previous reports documenting a demyelinating peripheral neuropathy in a complicated case of HSP.

The hereditary neuropathies, ataxias, and spastic paraplegias are groupings of genetically diverse disorders classified according to their predominant clinical phenotype. This case further extends the considerable overlap between these clinical syndromes.

\section{References}

1. Harding AE. Classification of the hereditary ataxias and paraplegias. Lancet 1983; 1: 1151-5.

2. Harding AE, Thomas PK. Peroneal muscular atrophy with pyramidal features. Journal of Neurology, Neurosurgery, and Psychiatry 1984; 47: 168-72.

3. Frith JA, McLeod JG, Nicholson GA, Yang F. Peroneal muscular atrophy with pyramidal tract features (hereditary motor and sensory type V) a clinical, neurophysiological and pathological study of a large kindred. Journal of Neurology, Neurosurgery, and Psychiatry 1994; 57: 1343-6.

4. Cross HE, McKusick VA. Troyer syndrome: a recessive form of spastic paraplegia with distal muscle wasting. Archives of Neurology 1967; 16: 473-85.

5. Silver JR. Familial spastic paraplegia with amyotrophy of the hands. Journal of Neurology, Neurosurgery, and Psychiatry 1966; 29: 135-44.

6. Thomas PK, Misra VP, King RHM, Muddle JR, Wroe S, et al. Autosomal recessive hereditary sensory neuropathy with spastic paraplegia. Brain 1994; 117: 651-9. 